\title{
Multi-agent Crisis Management in Transport Domain
}

\author{
Michał Konieczny, Jarosław Koźlak, and Małgorzata Żabińska \\ Department of Computer Science, \\ AGH University of Science and Technology \\ Al. Mickiewicza 30, 30-059 Kraków, Poland \\ \{kozlak,zabinska\}@agh.edu.pl, michal.t.konieczny@gmail.com
}

\begin{abstract}
A multi-agent system that solves static and dynamic versions of transport problem (Pickup and Delivery Problem with Time Windows) in presence of crises is shown here. Scenarios to serve different kinds of crises (especially vehicle failures and traffic jams) by agents are described. The results summarising system functioning for solving classical PDPTW as well as influence of crises and applied algorithms of serving them upon quality of obtained solutions have been presented.
\end{abstract}

\section{Introduction}

Efficient transport of people and goods is an important issue with different transport companies. For some branches of the industry costs of transport may reach even $70 \%$ of value of transported goods. Therefore it is justified to tend to minimise transport costs which influence the final price of goods and services.

Many transport problems may be treated as PDPTW, which is an extension of the widely examined Vehicle Routing Problem. Solving the PDPTW consists in finding routes which may be served by the minimal number of vehicles and to minimise the distance travelled. The distance is the total distance vehicles have to travel to serve the existing set of transport request between points of pick up and delivery, on the assumption that each request will be served within the time range defined by the time window, whereby the maximum capacity of vehicles is not exceeded. Since the problem has a large complexity (NP-problem), the research on solution of PDPTW concentrates rather on working out proper meta-heuristic methods than upon searching for exact solutions.

The purpose of the work carried out is to create the system which enables solving PDPTW with more accurate reflection of real conditions. PDPTW will be extended, among others, by a complex transport network, dynamics of transport requests as well as crises - unexpected events, which force changes of routes of transport teams. Objects, existing at the logistic firm, such as dispatching of tasks, vehicles, or being responsible for crisis situations service, may be intuitively implemented with the use of agents. 


\section{State of the Art}

\subsection{Transport Problems and Their Solving}

A frequently used approach to solve complex computational problems is to divide the task into two phases. In the first one, a relatively good primary solution is generated, whereas in the second phase, it is optimised. the following are popular construction heuristics which build primary solutions: Insertion Heuristic, Sweep Heuristic and Partitioned Insertion Heuristic described in [8]. Methods which improve the quality of obtained solution are based on multiple modifications of a set of routes with the use of local changes consisting in moving requests from the route (SPI - Single Paired Insertion), exchange of requests between routes (SBR - Swapping Pairs Between Routes) and change of sequence of requests for a given route (WRI - Within Route Insertion). The optimisation process may be managed using algorithms like tabu search [9, simulated annealing, evolutionary algorithms, Squeaky Wheel Optimisation [10] and Ant Colony optimisation [5].

\subsection{Multi-agent Solutions}

Agent systems which solve transport problems are based on two schemes: Contract Net [12] and simulated trading. An algorithm of iterative optimisation (Simulated Trading) [3] is used to improve the primary feasible solution. It applies a stock exchange mechanism, where vehicles optimise their plans by subsequent purchase and selling of transport requests. MARS [6] is an multi-agent system realising transport planning. Its purpose is to organise the cooperation between some transport companies, so as to serve the set of transport tasks in an optimal way.

TeleTruck is a system modelling process at the transport company. It is a holonic multi-agent system created by the firm DFKI [4]. TeleTruck has a richer functionality than MARS. It is a prototype of an application built with the essential help of a transport company. It plans real requests with the use of heterogenic agents modelling different vehicle forms. The main goal of TeleTruck is to model basic objects of the real world (drivers, trucks, trailers, containers) with the use of basic agents. These agents have a task to merge themselves and create holonic agents, cooperating together and performing tasks.

\subsection{Crises in Transport Systems}

The specific features of multi-agent systems, connected with decision making based on local, partial knowledge and dynamic system reorganisation in response to changes taking place, favour this approach when reacting to crisis situations. Here, we will especially focus on applications in the transport domain. In [11] a general idea of the model of such a system to react on crisis situations, prevent them and minimise consequences is given. The crisis situations analysed are most often delays caused by uncertain travel times and traffic jams [7. Furthermore, the presented MARS system was equipped with a module responsible for modelling and predicting traffic jams while constructing routes. 


\section{Concept of Multi-agent System}

The main task of the constructed system is to solve static and dynamic transport problems (PDPTW) and additionally recognising, serving and minimising disadvantageous results of the chosen types of crises situations. In such a kind of problem it is possible to distinguish units with different degrees of autonomy of action (dispatcher, vehicles), which cooperate to realise some common goals. This is the reason why an approach using multi-agent systems is applied. The environment, where the agents act is either the Euclidean space or a graph representing road connections.

\subsection{Agents' Description}

In the constructed system the following main types of agents exist: Dispatcher Agent, Execution Unit Agent (EUnit Agent) and Crisis Manager Agent.

\subsection{Dispatcher Agent}

Dispatcher Agent $A D$ plays a role of manager in the system. It controls the work and life cycle of Execution Unit agents, as well as it distributes transport requests between them in the best possible way. In the system only one Dispatcher Agent exists.

$$
A D=\left(G_{D}, K_{D}, A_{D}\right)
$$

where:

$G_{D}$ - agent's goal which is to minimise the function nv $+\mathrm{TD}$, where v - number of vehicles, TD -total distance, $\mathrm{n}$ - coefficient.

$K_{D}$ - agent's knowledge, $K_{D}=\{$ Reqs, StatReqs, Env, EnvStat $\}$, where Reqs - information about requests, StatReqs - information about status of requests, which assigns to each of them a value from the set \{received, rejected, allocated, picked-up, delivered, wait_trainshipment\}, Env — information about transport network, EnvStat - information about the current status of transport network,

$A_{D}$ - actions to be realised, $A_{D}=\{$ AquireEU, AllocateReq $\}$

\subsection{Execution Unit Agent}

The agent $A E U$ manages the transport unit which realises transport requests. Together with other units it participates in auctions of transport requests, and then it realises the assigned requests.

$$
A E U=\left(G_{E U}, P_{E U}, L o c, S_{E U}, K_{E U}, A_{E U}\right)
$$

where: 
$G_{E U}$ - agent's goal which is to realise the maximal possible number of requests

as well as minimising of the total distance,

$P_{E U}$ - planned travel route,

Loc - current agent's position,

$S_{E U}$ - agent's status, information about allocated requests,

$K_{E U}$ - agent's knowledge $K_{E U}=\{$ Reqs, Env, EnvStatg $\}$ comprising Reqs set of requests, Env - information about environment (transport network), EnvStat - information about status of environment taking into account information concerning traffic jams as well as parts of a network totally exluded from traffic,

$A_{E U}$ - actions of $A_{E} U=\{$ ReqPart, PickUp, Delivery, SendInfo\}, where ReqPart - participation in auctions of transport requests, PickUp - loading, Delivery - unloading, SendInfo - transfer of information about discovered crisis situations to Crisis Manager Agent.

\subsection{Crisis Manager Agent}

Crisis Manager Agent $A C M$ is dedicated to manage crisis situations originating in the system. The agent is responsible for the detection of crisis situations as well as handling them. Detection of the crisis situation may be done directly by ACM, or indirectly by information from another agent. Handling of crisis situations consists in such collaboration with other agents, so as to minimise all negative results of the situation. Currently, only one Crisis Management Agent exists in the system.

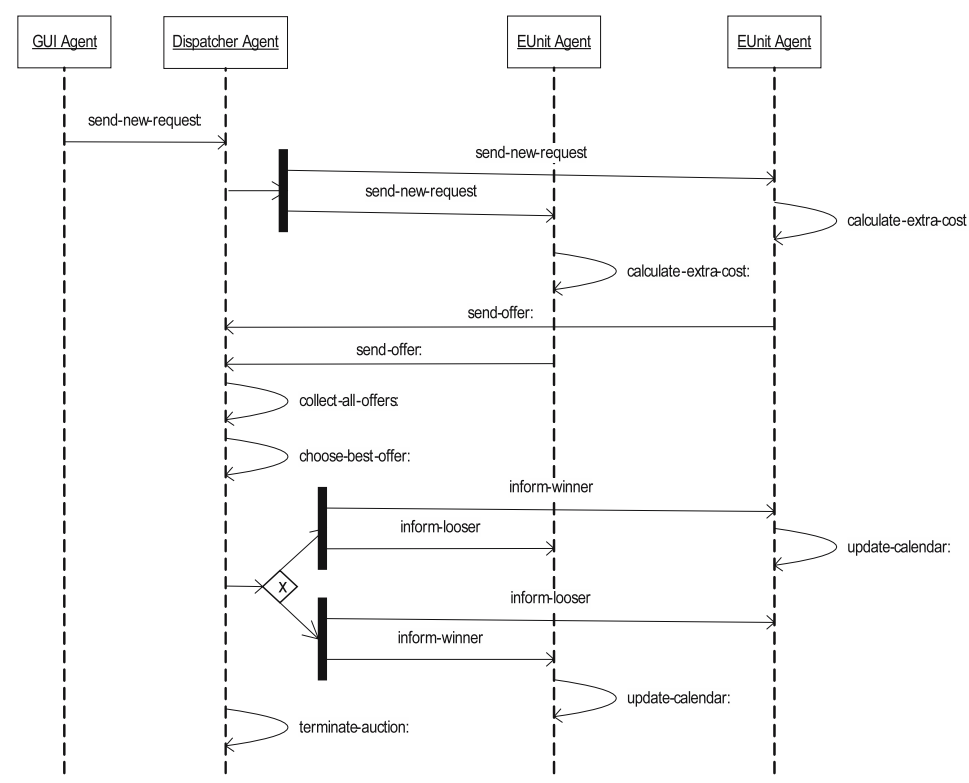

Fig. 1. Request allocation without crises handling 


$$
A C M=\left\{K_{A C M}, R_{A C M}, A_{A C M}\right\}
$$

where:

$K_{A C M}$ - knowledge about crisis situations

$R_{A C M}$ - decision rules describing actions to be undertaken, for a given crisis situation and situations occurring during its service

$A_{A C M}$ - actions performed by an agent, $A_{A C M}=\{$ SendInf, RecStat, RReal $\}$, SendInf - sending the information about crisis situation, ResStat - receiving information about the status of the crisis situation, RReal - proposal to reassign transport requests.

\subsection{Request Allocation Protocol}

In fig. 1, the request allocation to vehicles without crises handling is shown.

\section{Crises Handling}

Crisis situations are defined as any unpredictable events which have a significant impact on company activity. They include transport fleet faults, delays of receiving or delivering goods by customer, as well as unpredictable changes in road infrastructure, from intensive road traffic, which significantly increase the travel time, or faults of road sections. Occurrence of a crisis situation forces change of transport plan or forces the use of a larger amount of transport units.

\subsection{Types of Crises}

Crisis situations may be distinguished into three groups: crisis situations in transport requests, transport fleet and road infrastructure. The first group includes request withdrawal and delay in receiving commodity. The second group includes failures of the transport unit. The last group includes traffic jams and road blocks.

\subsection{Transport Unit Failure}

The discussed crisis situation involves temporary or permanent exclusion of a transport unit from use. Detection of this crisis situation involves Crisis Manager Agent "finding out" at first about the occurrence of this crisis situation. Next, Crisis Manager Agent informs transport unit about the situation occurred. In cases when the expected time of repair of the defective transport unit is short enough to let all planned requests be realised in given time windows, then the crisis situation does not significantly affect the course of simulation. It only extends the total time of realising the whole transport plan. However, if a failure of the transport fleet unit is serious, it must be replaced with an adequate vehicle, and transport requests in its transport plan must be passed on to other transport units. Requests may be transferred on to other transport 


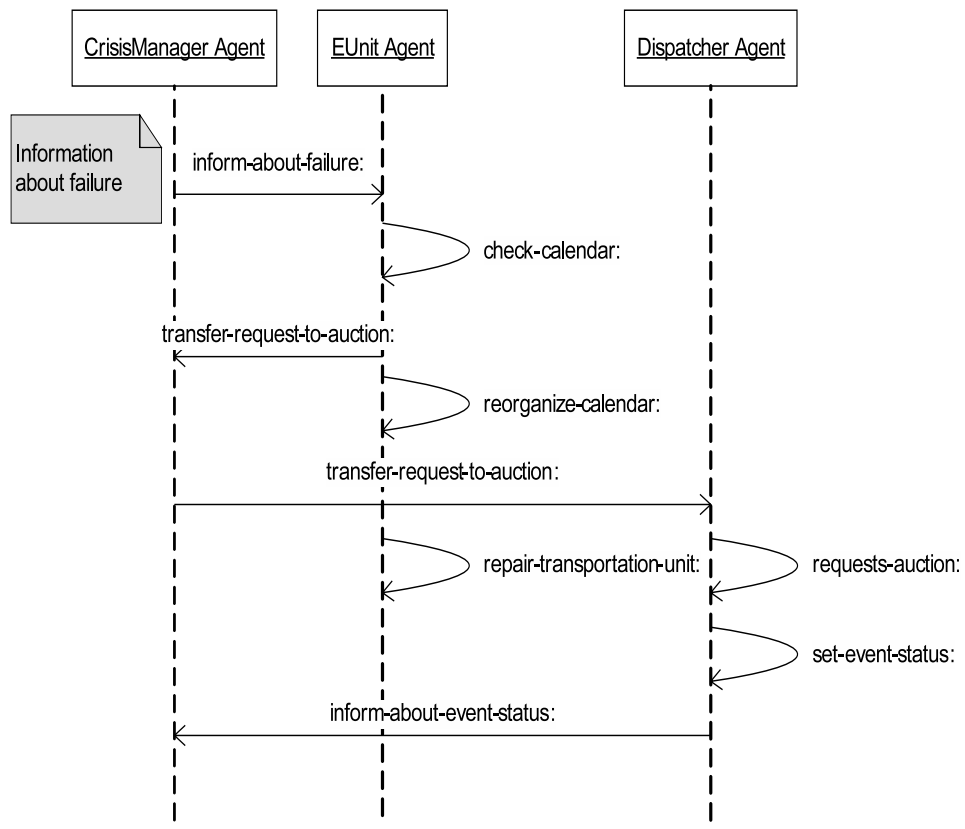

Fig. 2. Interactions: Handling of vehicle failure

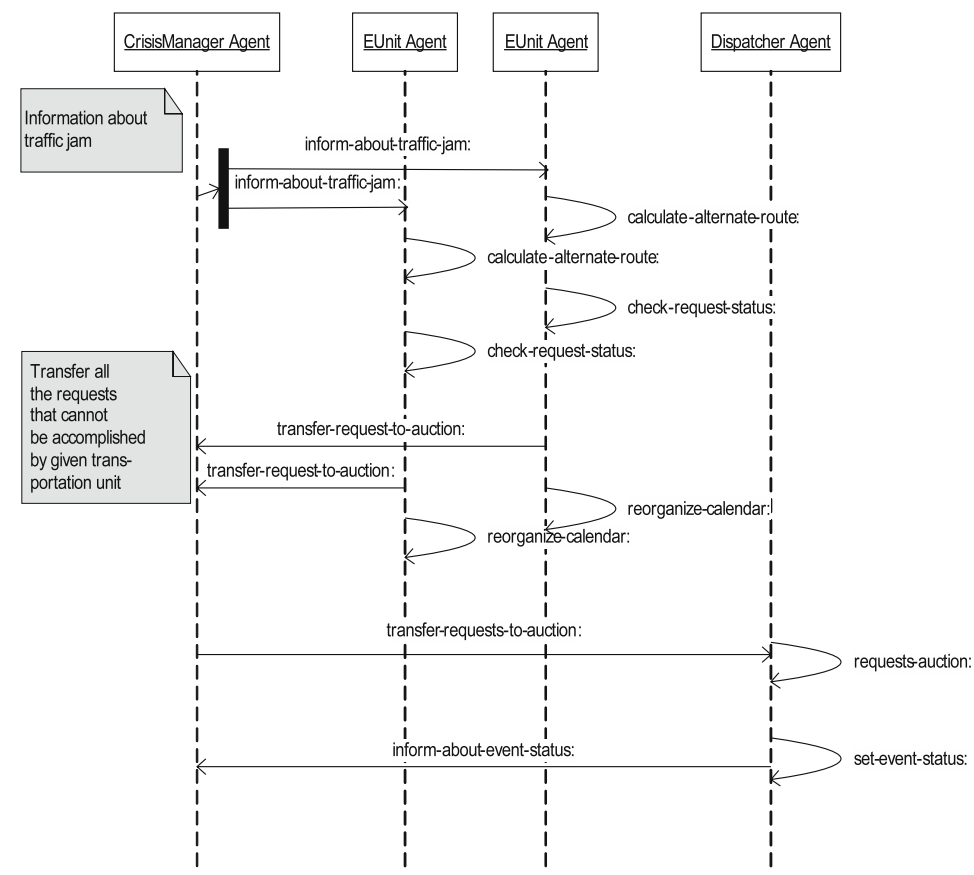

Fig. 3. Interactions: Handling of traffic jam 
units. The faulty unit must be transported to a point of repair, which increases the cost of realising the transport plan. In cases when the transport set failure is serious (long time of repair), it is necessary to put the next transport requests in the transport plan up for auction, so that the time of realising them closes in a definite time window.

\subsection{Traffic Jam}

Traffic jams have significant influence on shipping agent activity. Long-lasting jams contribute to delays in realising the transport requests in given time windows. Detection of a crisis situation such as a traffic jam may be performed in two ways. (1) A transport set moving along a section of the transport network, on which a traffic jam occurs, informs Crisis Manager Agent about this event. (2) Crisis Manager Agent can receive information about this event directly from the crisis situations generator. After the traffic jam has been detected, there are three alternative scenarios presented in fig. 3. depending on the certain situation. (1) If the delay resulting from a traffic jam has no influence on realising the further transport plan by a transport unit, the unit should continue without any changes. (2) If the delay results in a subsequent request in the transport plan to be delayed, the transport plan should be changed, to avoid this kind of situation. The change of the plan involves determining alternative routes, bypassing the section with traffic jam (3) If, despite determining an alternative route, subsequent request realisations are delayed, they should be passed on to other transport units.

\section{Realisation}

The system architecture scheme is presented in fig. 4. JADE platform (Java Agent Development Framework) version 3.3 [2] has been used. Fig. 4 presents all

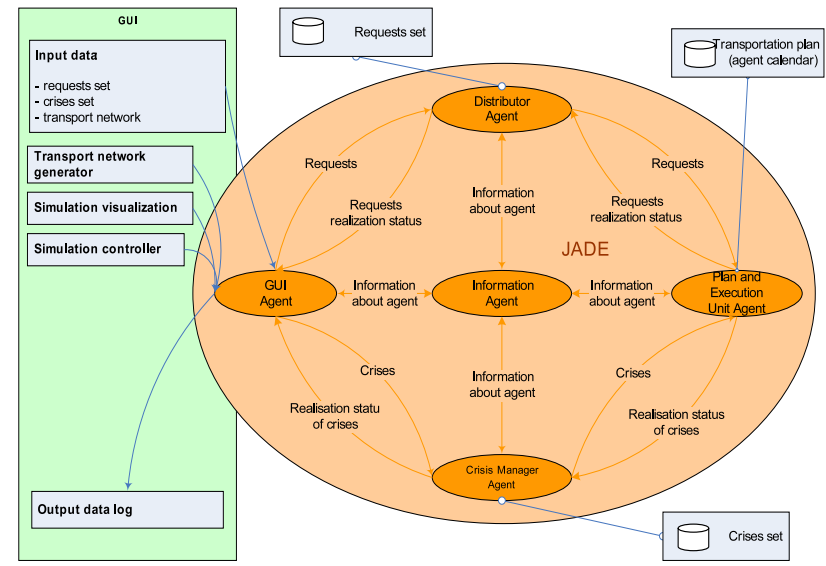

Fig. 4. System architecture 
types of agents existing in the system and JADE platform range. It also presents data stored by certain agents during the simulation. In Dispatcher Agent's case, it constitutes a set of transport request to be realised, as well as information considering the status of their performance. Each agent of the Planning and Executive Units, has a transport plan. A set of crisis situations to serve together with their status, is stored by Crisis Manager Agent.

Transport network generator delivers environment for agents in the system.

\section{Experiments and Results}

The input data for the system may be distinguished into three categories. The first concerns transport requests, the second - road networks, connected points of pickup or delivery and dispatch centre as well as connections between them, and the third concerns crisis situations.

Evaluation of the algorithms applied to solve PDPTW was done on the basis of benchmarks, which contain sets of transport requests [1], developed by Li and Lim [9]. The published sets of tests belong to the three kinds of problems: $\mathrm{C}-$ locations placed in clusters, $\mathrm{R}$ - locations placed randomly or $\mathrm{RC}$ - locations placed either in clusters or randomly. Additionally, each kind of problems may have small or large time windows and may have different numbers of requests to be served.

\subsection{Solving of PDPTW Static Problems}

In this section selected results of solving PDPTW for static and dynamic cases are described. In fig. 5 the average number of used vehicles and the average total distances for selected groups of benchmarks are presented. The results obtained by the created algorithm are not much worse than the best known solutions [1].

The following experiments lead in the direction of using the maps of transport roads generated according to prepared algorithms considering requests points from Li \&Lim benchmarks and an analysis of the dynamic version of the problem.

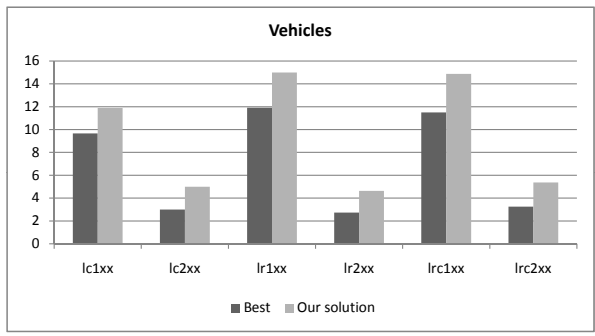

a)

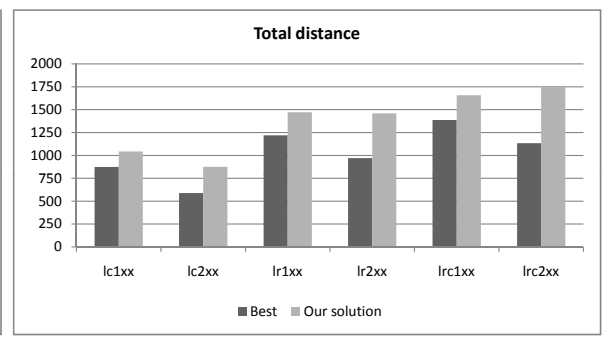

b)

Fig. 5. Results: Static PDPTW: a)Vehicles needed b)Total travel distance 


\subsection{Crises}

The results will be evaluated by the number of used vehicles and the total travel distance as well their increase in comparison to the experiments without crisis situations. In each of the performed tests 10 crises situations of each analysed kind have been randomly generated. After performing the series of tests, numbers of non-performed requests for the given kinds of crises situations are: $10(19.4 \%)$ for request withdrawal, $2.1(4.1 \%)$ for the delay of the delivery/receipt of the package by customer, $13,7(26.6 \%)$ for vehicle breakdown, $4.6(8.9 \%)$ for the increase of the travel time caused by traffic jams and $5.2(10.1 \%)$ for closed routes.

In fig. 6 $\mathrm{b}$ the number of used vehicles, the average for each group of request, during the realisation of requests with different kinds of crisis situations introduced are shown. In the vertical axis the number of vehicles used is presented and in the additional vertical axis on the right the same value in comparison to the number of vehicles used during the realisation of requests without crisis situations is shown. In fig. $6 \mathrm{~b}$ the average distances travelled by all vehicles, for each group of requests during the realisation of requests with different kinds of crisis situations introduced are presented. In the vertical axis the distance travelled by vehicles of the fleet is presented and on the right side of the figure, the same value in comparison to the distance travelled by the vehicles, during the realisation without crisis situations is shown.

The largest decrease of the number of performed requests and the increase of the used vehicles took place for the cases with vehicle failures and road closures. The results for problems with crises such as customer delay and increase of travel time are burdened by relatively low additional costs. The increase of travel times and closing of routes especially lengthened the total distance because it forced a search for other, longer routes.

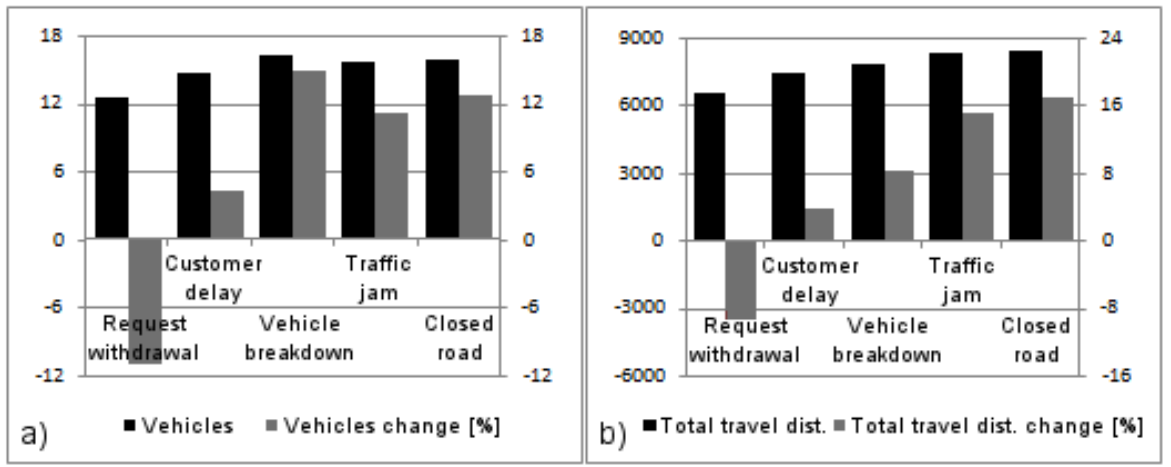

Fig. 6. Results for the different kinds of crises: a) Vehicles needed b)Total travel distance 


\section{Conclusions}

During this work, a multi-agent system for solving transport problem PDPTW placed under the real conditions and with features of solving several kinds of potential crisis situations has been realised.

Future work will focus on the improvement of the quality of optimisation algorithms, introduction of soft time windows and the applications of holons. The soft time windows will make it possible to build plans which take into consideration fees dependent on the size of the delay. The application of holonic approach makes it possible to take into consideration the transport units composed of different components so as to be best adjusted to needs.

\section{References}

1. Benchmarks - Vehicle Routing and Travelling Salesperson Problems, http://www.sintef.no/static/am/opti/projects/top/

2. Java Agent DEvelopment Framework, http://jade.tilab.com/

3. Bachem, A., Hochstattler, W., Malich, M.: Simulated Trading A New Parallel Approach for Solving Vehicle Routing Problems. In: Proceedings of the International Conference Parallel Computing: Trends and Applications (1994)

4. Burckert, H.-J., Fischer, K., Vierke, G.: Transportation scheduling with holonic MAS - the TELETRUCK approach. In: Third International Conference on Practical Applications of Intelligent Agents and Multiagents, PAAM 1998 (1998)

5. Doerner, K., Hartl, R.F., Reimann, M.: Ant Colony Optimization applied to the pickup and delivery problem. Technical Report Working Paper 76, Departament of Managment Science, University of Vienna, Wien, Austria (November 2000)

6. Fischer, K., Muller, J., Pischel, M.: Cooperative Transportation Scheduling: an Application Domain for DAI. In: Applied Artificial Intelligence, pp. 1-33 (1996)

7. Koźlak, J.: Learning in cooperating agents environment as a method of solving transport problems and limiting the effects of crisis situations. In: Shi, Y., van Albada, G.D., Dongarra, J., Sloot, P.M.A. (eds.) ICCS 2007. LNCS, vol. 4488, pp. 872-879. Springer, Heidelberg (2007)

8. Lau, H., Liang, Z.: Pickup and Delivery with Time Windows: Algorithms and Test Case Generation. In: Proceeedings of 13th IEEE International Conference on Tools with Artificial Intelligence, ICTAI 2001 (2001)

9. Li, H., Lim, A.: A Metaheuristic for the Pickup and Delivery Problem with Time Windows. In: Proceedings of 13th IEEE International Conference on Tools with Artificial Intelligence (ICTAI 2001), Dallas, USA (2001)

10. Lim, H., Lim, A., Rodrigues, B.: Solving the Pick up and Delivery Problem using "Squeaky Wheel" Optimization with Local Search. In: Proceedings of American Conference on Information Systems, AMCIS 2002, USA (2002)

11. Nawarecki, E., Koźlak, J., Dobrowolski, G., Kisiel-Dorohinicki, M.: Discovery of crises via agent-based simulation of a transportation system. In: Pěchouček, M., Petta, P., Varga, L.Z. (eds.) CEEMAS 2005. LNCS, vol. 3690, pp. 132-141. Springer, Heidelberg (2005)

12. Smith, R.G.: The contract net protocol: high-level communication and control in a distributed problem solver. IEEE Transactions on Computer, 1104-1113 (December 1980) 